\title{
Diversidad y estado de conservación de la mastofauna terrestre del municipio de San Vicente del Caguán, Caquetá, Colombia
}

\section{Diversity and conservation state of land mammals of the municipality of San Vicente del Caguán, Caquetá, Colombia}

\author{
Alejandra Niño-Reyes*, Alexander Velásquez-Valencia*
}

\section{Resumen}

\begin{abstract}
Objetivos: Determinar la diversidad y el estado de conservación de los mamíferos terrestres en el municipio de San Vicente del Caguán. Metodología: La investigación se realizó en 4 parches de vegetación caracterizados por bosque denso alto de tierra firme, de galería, vegetación secundaria y pastos enmalezados rodeados por pastos limpios. Se empleó la técnica de muestreos visuales en recorridos diurnos para el registro de mamíferos arborícolas y no voladores; para la captura de murciélagos se instalaron 4 de redes por parche de manera ad libitum, entre las 17:30 hasta las 23:00 horas. Los datos obtenidos fueron analizados mediante una curva de acumulación, índices de diversidad de Margaleft, Shanon $\mathrm{H}$, Simpson, Dominancia y Equitatividad, análisis de similitud y de correspondencia. Resultados: Se registraron 105 individuos de mamíferos, distribuidos en ocho órdenes, 15 familias y 23 especies. Se obtuvo la mayor diversidad $(\mathrm{H})$ y la mayor riqueza $(\mathrm{M})$ en parches de bosque alto de tierra firme rodeada por pastos y vegetación secundaria y la mayor equitatividad en bosque de galería rodeado por pastos limpios. Se identificaron tres especies de especial interés para la conservación, Tapirus terrestris, Ateles belzebuth y Lagothrix lagothricha. Conclusiones: El área de estudio corresponde a una zona de alta diversidad de mamíferos, con remanentes de bosque que constituyen importantes áreas de refugio para la mastofauna. Es indispensable generar iniciativas de conservación porque las condiciones de presión (caza, la extracción de madera y explotación petrolera) ponen en constante riesgo el mantenimiento de esta comunidad en el departamento del Caquetá.
\end{abstract}

Palabras claves: Categorías de amenaza, Caquetá, Mastofauna, Riqueza de especies.

\begin{abstract}
Objective: Determine the diversity and conservation status of land mammals of the municipality of San Vicente del Caguán, Caquetá, Colombia. Methodology: The research was conducted in 4 vegetation patches of characterized by high dense forest od solid land, gallery forest, secondary vegetation and weedy grasses surrounded by clean pasture. Visual sampling technique was used in day runs for recording arboreal mammals and flightless; for bats catching 4 mist nets were installed in each patch by ad libitum way, between 17:30 to 23:00. The obtained information was analyzed by an accumulation curve, Margaleft's diversity index, Shanon $\mathrm{H}$, Simpson, Dominance and evenness, similarity and correspondence analysis. Results: 105 individuals of mammals, distributed in eight orders, 15 families and 23 species were recorded. the greatest diversity $(H)$ and richest $(M)$ was founded in high dense forest of solid land patches surrounded by grasses and secondary vegetation and greater evenness in Gallery Forest surrounded by clean pasture. Three species of special interest for conservation were identified, Tapirus terrestris, Ateles belzebuth y Lagothrix lagothricha. Conclusions: The study area is a place of high mammals diversity, with forest remaining shelter which are important areas for mammals. It is so important to make conservation initiatives, because stress conditions (hunting, wood and oil extraction) are dangerous for the maintenance of this community in the department of Caquetá.
\end{abstract}

Keywords: Caquetá, Mammals, Species richness, Threatened categories.

* Centro de Investigación de Biodiversidad Andino-Amazónica, Museo de Historia Natural, Universidad de la Amazonia, Grupo Fauna Silvestre, Sede Centro, Florencia, Caquetá, Colombia. e-mail: alejandra.vtab@gmail.com alexandervelasquezvalencia@gmail.com Fecha recepción: Febrero 4, 2016 Fecha aprobación: Mayo 12, 2016 


\section{Introducción}

La diversidad de mamíferos presente en Colombia representa $11.8 \%$ de la mastofauna mundial, con 518 especies (Ramírez-Chaves et al. 2016); su estudio es indispensable para la comprensión de escalas macroecológicas, áreas de distribución y condiciones de calidad de los ecosistemas porque por sus requerimientos de hábitat y la sensibilidad que presentan a los cambios del paisaje se han considerado algunas especies de estos grupos como bioindicadoras (Rodríguez-M et al. 2006). La información relacionada con la diversidad biológica constituye una herramienta con características específicas de un espacio y tiempo determinado (Villarreal et al. 2004) que reviste importancia para la planificación de investigaciones que permitan identificar cambios en los ecosistemas y delimitar áreas prioritarias para la conservación (Boddicker et al. 2002).

Zonas como el departamento del Caquetá no cuentan con investigaciones de mastofauna disponibles que permita actualizar la información, conocer el estado de las poblaciones y la distribución de este grupo. Actualmente se conocen listados nacionales de especies de mamíferos (Solari et al. 2013) que relacionan la mastofauna presente en el departamento y algunos estudios de grupos específicos como quirópteros (Marín-Vázques y Aguilar-Gonzáles 2005a, Marín-Vázques et al. 2005b, Gonzáles-Reyes y Velásquez-Valencia 2011) y marsupiales (García-Cedeño et al. 2005).

Actualizar esta información y conocer la distribución real de los mamíferos de la Amazonia Andina en el departamento del Caquetá es fundamental debido a la acelerada reducción y pérdida de hábitat. En el departamento se extraen cerca de $35.231 \mathrm{~m}^{3}$ de madera por año, por consecuencia de procesos de intervención forestal y agrícola, actividades como la minería, explotación petrolera y cultivos con la finalidad de sintetizar sustancias alucinógenas (IGAC 2014). Estas acciones ponen en riesgo la disponibilidad de los recursos y los servicios ecosistémicos, reduciendo conectividad del paisaje y con ello la movilidad de las especies (Santos y Tellería 2006). La pérdida de la diversidad generada por estas actividades, no ha sido cuantificada; el problema se agudiza aún más, porque no se cuenta con estudios de fauna específica en los bosques de la Amazonia Andina colombiana que permitan realizar diagnósticos que soporten programas para su conservación.

En este sentido, el siguiente estudio se realizó en el municipio de San Vicente del Caguán, en un ecosistema de la Amazonia Andina, ubicado en la intersección de la región andina, la región amazónica y la sierra de la Macarena en Colombia. Este trabajo evidencia la diversidad de especies, su importancia ecosistémica, las elevadas categorías de amenaza en las que se encuentran algunas de ellas y los factores limitantes para la investigación en esta zona del país.

\section{Metodología}

Área de estudio. El estudio se realizó en la vereda Los Pozos, municipio de San Vicente del Caguán, departamento del Caquetá, Colombia, ubicado en zona geográfica de la Amazonia Andina colombiana. El departamento cuenta con una superficie de 88,965 $\mathrm{km}^{2}$, representando $7,8 \%$ del territorio nacional, área que corresponde a una franja entre la Amazonia y la Orinoquía. Esta región presenta una temperatura promedio anual que fluctúa entre $22^{\circ} \mathrm{C}$ y $24^{\circ} \mathrm{C}$, con precipitaciones superiores a $3.000 \mathrm{~mm}$ anuales (IGAC 2014). El paisaje de la región se caracteriza por ser un mosaico de coberturas vegetales que han sido objeto de acelerados procesos de fragmentación (IGAC 1993).

Métodos. Se seleccionaron cuatro parches de bosque, teniendo en cuenta actividades antrópicas en una matriz de bosque rápidamente modificado. Estos correspondían a bosque denso alto de tierra firme rodeado por pastos enmalezados y vegetación secundaria $\left(\mathrm{BAT} / \mathrm{PV}_{1}\right)$, bosque de galería y pastos enmalezados rodeados por pastos limpios $\left(\mathrm{BG} / \mathrm{P}_{2}\right)$, bosque de galería y pastos enmalezados con un relicto de vegetación secundaria rodeado por pastos limpios $\left(\mathrm{BG} / \mathrm{VP}_{3}\right)$ y bosque denso alto de tierra firme en una matriz de pastos limpios (BAT/ $\mathrm{P}_{4}$ ) (Figura 1).

Fase de campo. Se empleó la técnica de muestreos visuales (visual encounter sourvey (VES) (Crump y Scott 1994) en recorridos diurnos y nocturnos, tomando registro escrito de vocalizaciones y fotográfico de los mamíferos arborícolas y rastros que se encontraron en cada uno de los parches, entre los meses de agosto de 2014 y febrero de 2015. Para la captura de murciélagos se instalaron cuatro redes de niebla de $12 \times 2,5 \mathrm{~m}$ y ojo de malla de $12 \mathrm{~mm}$, en cada 


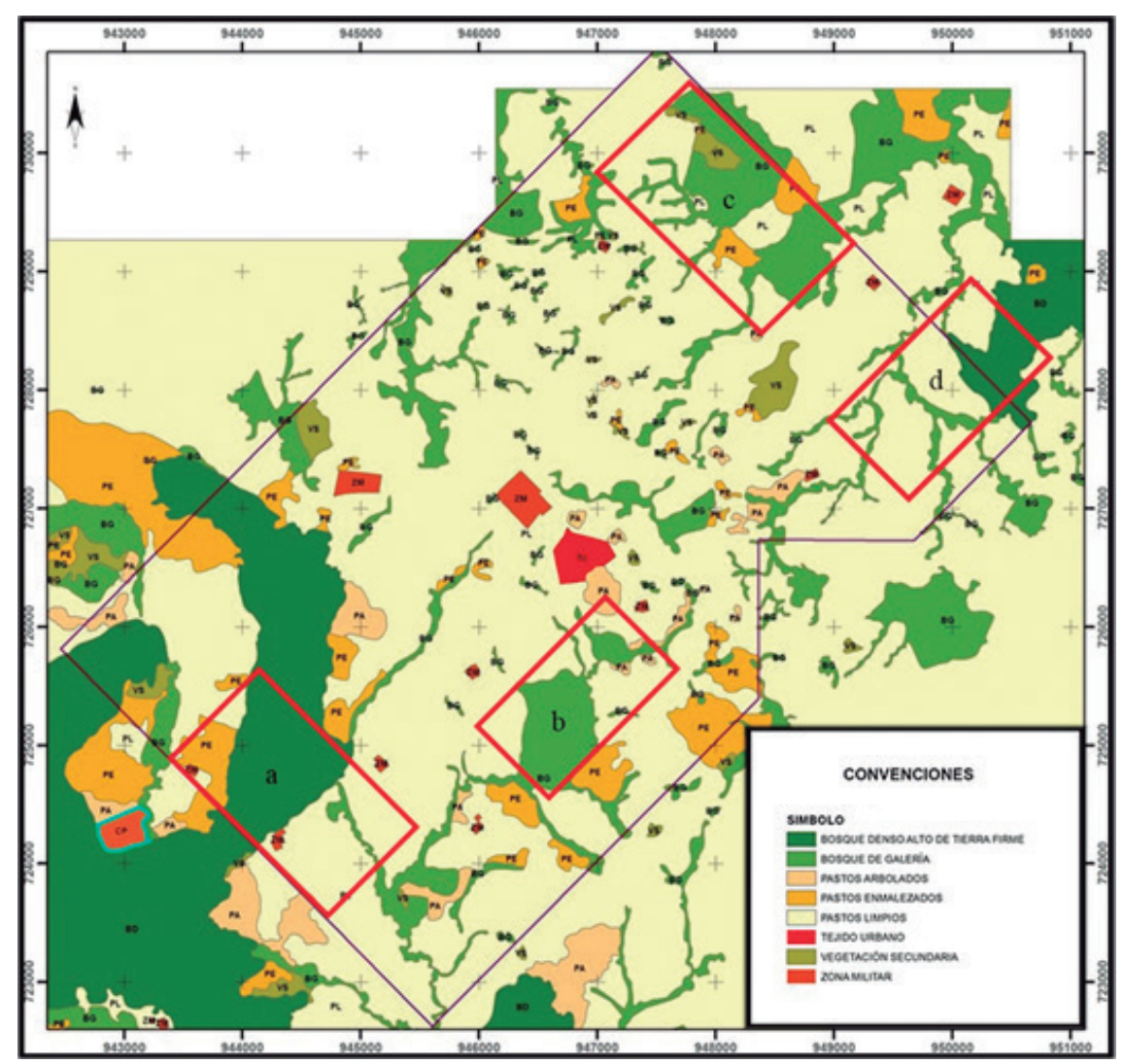

Figura 1. Mapa descriptivo de las coberturas vegetales de los parches de bosque del área de estudio en el sector Los Pozos del municipio de San Vicente del Caguán, Caquetá, Colombia.

a. Bosque denso alto de tierra firme rodeado por pastos enmalezados y vegetación secundaria $\left(\mathrm{BAT}_{\mathrm{PV}}\right.$ ), b. Bosque de galería y pastos enmalezados rodeados por pastos limpios $\left(B G / P_{2}\right)$, c. Bosque de galería y pastos enmalezados con un relicto de vegetación secundaria rodeado por pastos limpios $\left(\mathrm{BG} / \mathrm{VP}_{3}\right)$ y d. Bosque denso alto de tierra firme en un matriz de pastos limpios $\left(\mathrm{BAT} / \mathrm{P}_{4}\right)$.

uno de los parches de manera ad libitum; las capturas de murciélagos se realizaron entre las 17:30 hasta las 23:00 horas con revisiones cada 30 min período de mayor actividad para este orden.

Análisis de datos. Para los transectos de observación el esfuerzo de muestreo fue medido en número de horas totales de detección (visual y auditiva) por distancia total recorrida, teniendo en cuenta los datos de la distancia de cada trayecto, la hora de inicio y de finalización de cada recorrido. El éxito de observación fue determinado al dividir el número de observaciones o registros entre el esfuerzo de muestreo. En la captura de murciélagos el esfuerzo de muestreo fue medido en horas-red, donde una hora-red equivale a una red de $12 \times 2 \mathrm{~m}$ (en este muestreo) abierta durante una hora. Este cálculo se realizó para cada parche, también se calculó el esfuerzo de muestreo acumulado para toda el área.

Se estimó la representatividad de las especies mediante la elaboración de curvas de acumulación y la comparación con los valores de riqueza máximos esperados a partir de las funciones de Cole Rarefraction, Chao 1, Chao 2 y Jack 1, realizadas por el método de rarefacción mediante el programa EstimateS Versión 9.1.0. Este método calcula un valor esperado de especies en diferentes tamaños de muestra. Para el análisis de diversidad se utilizaron los índices de Simpson-D, Margaret, Shannon H, Dominance D y Equitabilty J y así evaluar la variabilidad en la composición de cada uno de los parches.

Los mamíferos fueron identificados por medio de las guías ilustradas de Mamíferos Grandes de Loreto Perú (Leite 2012), Primates de Colombia (Defler 2004) y clave de campo para identificación de los murciélagos de Bolivia (Aguirre et al. 2009). Los datos obtenidos fueron incluidos en una base de datos con las cuales se elaboraron matrices y listas de especies registradas en cada parche. Se evaluó la relación entre los parches con base en las abundancias por medio de un análisis de similitud. 


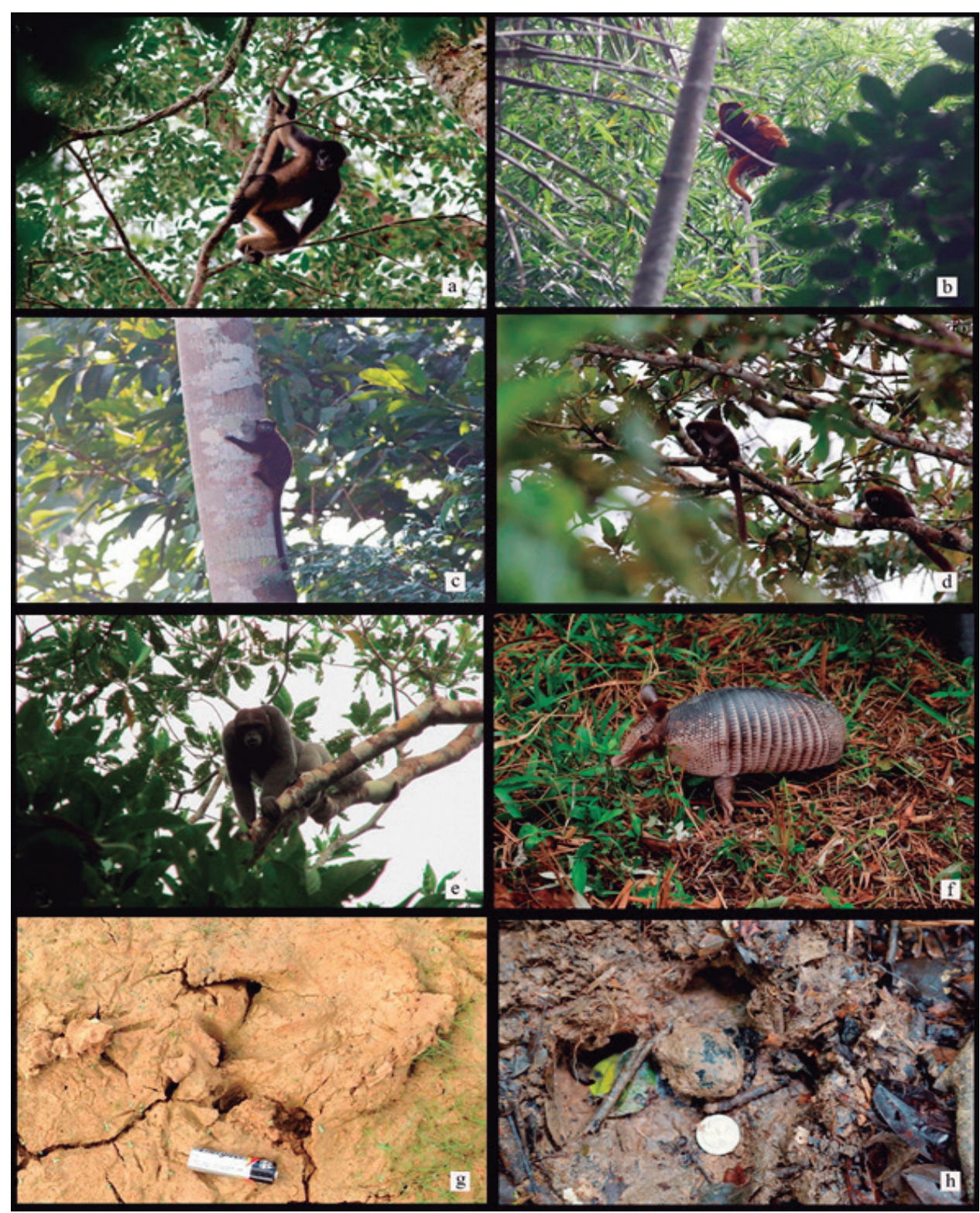

Figura 2. Fotografías de registros de mamíferos encontrados en el área de estudio. a. Ateles belzebuth, b. Alouatta seniculus, c. Leontocebus nigricolis, d. Cheracebus torquatus, e. Lagothrix lagothricha lugens, f. Dasypus novemcinctus, g. Huella de Hydrochoerus hydrochaeris, h. Huella de Tapirus terresstris.

Para le determinación del estado de conservación se realizaron revisiones en listados nacionales como la Resolución 192 del 2014 del Ministerio del Medio Ambiente y Desarrollo Sostenible (Rodríguez et al. 2006) (las especies silvestres amenazadas de la diversidad biológica colombiana), Convención sobre el Comercio Internacional de Especies Amenazadas de Fauna y Flora Silvestres (apéndices CITES) y a nivel internacional, la Unión Internacional para Conservación de la Naturaleza (UICN) Versión 2016-2. Para la identificar la afinidad de las especies amenazadas con los parches se realizó un análisis de correspondencia en XLSTAT Versión de Prueba 9.0.

\section{Resultados}

La diversidad de mamíferos está compuesta por 105 individuos, distribuidos en 8 órdenes, 15 familias y 23 especies (Tabla 1, Figura 2). El orden más rico fue Primates dominando en todos los parches. De las 15 familias, las más ricas fueron las Phyllostomidae con cinco especies y Cebidae con cuatro, las demás 
Tabla 1. Listado de especies de mamíferos presentes en la vereda los Pozos, San Vicente del Caguán y vegetación asociada

\begin{tabular}{|c|c|c|c|c|}
\hline Clase Mammalia & BAT/PV $_{1}$ & $\mathrm{BAT} \mathrm{PV}_{2}$ & $\mathrm{BAT} / \mathrm{PV}_{3}$ & BAT/PV $_{4}$ \\
\hline \multicolumn{5}{|l|}{ Orden Artiodactyla } \\
\hline \multicolumn{5}{|l|}{ Familia Tayassuidae } \\
\hline Pecari tajacu (Linnaeus, 1758) & & & $x$ & \\
\hline \multicolumn{5}{|l|}{ Orden Carnivora } \\
\hline \multicolumn{5}{|l|}{ Familia Canidae } \\
\hline Cerdocyon thous (Linnaeus, 1766) & & & $x$ & \\
\hline \multicolumn{5}{|l|}{ Orden Chiroptera } \\
\hline \multicolumn{5}{|l|}{ Familia Phyllostomidae } \\
\hline Carollia brevicauda (Schinz, 1821) & $x$ & $x$ & $x$ & \\
\hline Carollia perspicillata (Linnaeus, 1758) & $x$ & & & \\
\hline Rhinophylla pumilio Peters, 1865 & & $x$ & & \\
\hline Sturnira sp. Gray, 1842 & $x$ & & & \\
\hline \multirow{2}{*}{\multicolumn{5}{|c|}{ Familia Vespertilionidae }} \\
\hline & & & & \\
\hline Myotis sp. Kaup, 1829 & $x$ & & & \\
\hline \multicolumn{5}{|l|}{ Orden Cingulata } \\
\hline \multicolumn{5}{|l|}{ Familia Dasypodidae } \\
\hline Dasypus novemcinctus Linnaeus, 1758 & $x$ & & $x$ & $x$ \\
\hline \multicolumn{5}{|l|}{ Orden Didelphimorphia } \\
\hline \multicolumn{5}{|l|}{ Familia Didelphidae } \\
\hline Didelphis marsupialis Linnaeus, 1758 & & $x$ & $x$ & \\
\hline \multicolumn{5}{|l|}{ Orden Perissodactyla } \\
\hline \multicolumn{5}{|l|}{ Familia Tapiridae } \\
\hline Tapirus terrestris (Linnaeus, 1758) & & & $x$ & \\
\hline \multicolumn{5}{|l|}{ OrdenPrimate } \\
\hline \multicolumn{5}{|l|}{ Familia Aotidae } \\
\hline Alouatta seniculus (Linnaeus, 1766) & & $x$ & $x$ & \\
\hline \multicolumn{5}{|l|}{ Familia Atelidae } \\
\hline Ateles belzebuth É. Geoffroy, 1806 & & $x$ & & $x$ \\
\hline Lagothrix lagothricha Humboldt, 1812 & $x$ & & $x$ & $x$ \\
\hline \multicolumn{5}{|l|}{ Familia Cebidae } \\
\hline Cheracebus torquatus (Hoffmannsegg, 1807) & $x$ & $x$ & & \\
\hline Leontocebus nigricollis (Spix, 1823) & $x$ & & & \\
\hline Saimiri sciureus (Linnaeus, 1758) & $x$ & & & $x$ \\
\hline Sapajus apella (Linnaeus, 1758) & $x$ & $x$ & & $x$ \\
\hline \multicolumn{5}{|l|}{ Orden Rodentia } \\
\hline \multicolumn{5}{|l|}{ Familia Dasyproctidae } \\
\hline Dasyprocta sp. Illiger, 1811 & & & $x$ & \\
\hline \multicolumn{5}{|l|}{ Familia Eritozontidae } \\
\hline Coendou sp. Lacépède, 1799 & $x$ & & & \\
\hline \multicolumn{5}{|l|}{ Familia Hydrochaeridae } \\
\hline Hydrochoerus hydrochaeris (Linnaeus, 1766) & & & & $x$ \\
\hline \multicolumn{5}{|l|}{ Familia Proechimidae } \\
\hline Proechimys sp. J.A. Allen, 1899 & & & $x$ & \\
\hline Familia Sciuridae & & & & $x$ \\
\hline Hadrociurus igniventris (Wagner, 1842) & & & $x$ & $x$ \\
\hline
\end{tabular}

BAT/PV1: bosque denso alto de tierra firme rodeado por pastos enmalezados, BG/P2: vegetación secundaria bosque de galería y pastos enmalezados rodeados por pastos limpios, BG/VP3: bosque de galería y pastos enmalezados con un relicto de vegetación secundaria rodeado por pastos limpios, BAT/P4, bosque denso alto de tierra firme en un matriz de pastos limpios.

familias a excepción de Atelidae presentaron una especie. En total se realizaron 36 puntos de observaciones de los cuales solo 19 puntos fueron efectivos para la detección de la presencia de especies de mamíferos.

Según los estimadores de Chao 1, Chao 2 y Jack 1 (Figura 3) el esfuerzo de muestreo está representa- 


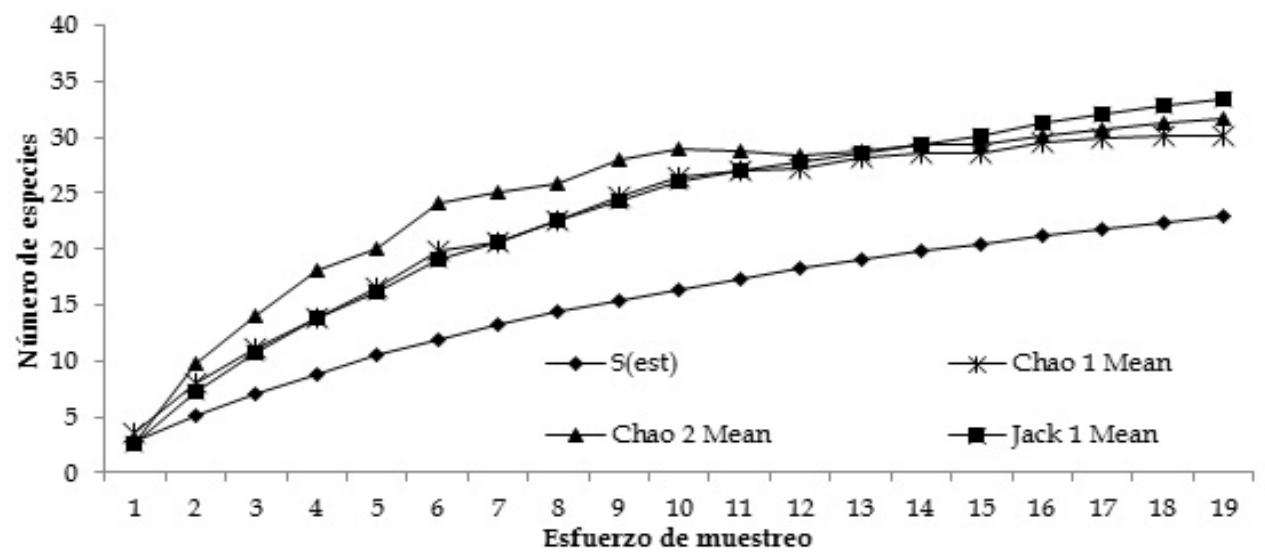

Figura 3. Curva de colector de las especies de mamíferos presentes. Cada esfuerzo de muestreo corresponde a punto de observación de radio de 50 m en un período de observación de 15 minutos.

do en 23 especies, que corresponden a más de $70 \%$ de las especies esperadas teniendo en cuenta que la curva no alcanza la asíntota, con una tendencia de incremento en la pendiente. El esfuerzo de captura fue de 160 horas/red, con un esfuerzo activo de 1920 horas/hombre de observación directa, $1080 \mathrm{~h} / \mathrm{h}$ de observaciones puntuales y $840 \mathrm{~h} / \mathrm{h}$ a búsquedas intensiva en los 4 parches.

La abundancia de mastofauna a nivel de parche fue mayor en el BAT/PV $\mathrm{PV}_{1}$ dominada por Carollia brevicauda y la más baja se registró en el $\mathrm{BAT} / \mathrm{P}_{4}$ dominada por Saimiri sciureus. El BAT/PV fue el más rico con 12 especies seguido por el $\mathrm{BG} / \mathrm{VP}_{3}$ con 11 y el parche $\mathrm{BG} / \mathrm{P}_{2}$ y BAT $/ \mathrm{P}_{4}$ con 7 respectivamente.

Diversidad de mamíferos. La mayor diversidad (H) y la mayor riqueza (M) se presentó en el BAT/ $\mathrm{PV}_{1}$ con 38 individuos distribuidos en 12 especies. La mayor equitatividad y el segundo parche con mayor $\mathrm{H}$ y $\mathrm{M}$ fue el $\mathrm{BG} / \mathrm{P}_{2}$, con un registro de 28 individuos correspondientes a once especies. El parche con menor abundancia y riqueza $(\mathrm{S})$, menor $\mathrm{H}$ y mayor dominancia (D) fue el BAT/ $\mathrm{P}_{4}$ (Tabla 2).

El área de estudio mostró heterogeneidad (Figura 4); en el dendrograma se evidencian dos agrupamientos: 1) de los parches $\mathrm{BAT} / \mathrm{PV}_{1}$ y $\mathrm{BAT} / \mathrm{P}_{4}$ con un porcentaje de similitud del $26,7 \%$ y 2) parches $\mathrm{BG} / \mathrm{P}_{2}$ y $\mathrm{BG} / \mathrm{VP}_{3}$ con el $20 \%$. Los parches $\mathrm{BAT} / \mathrm{PV}_{1}$ $\mathrm{yBG} / \mathrm{VP}_{3}$ muestran especies exclusivas mientras que los demás varían de 3 a 1 (Tabla 3). Asimismo, se observa en la Figura 5 que en su primer eje agrupa y genera un gradiente de complejidad con los parches de mayor heterogeneidad como BAT/PV 1 y BG/ $\mathrm{P}_{2}$, en estos se asocian especies como Cheracebus tor- quatus, Carollia brevicauda, Leontocebus nigricollis y en el extremo positivo el $\mathrm{BG} / \mathrm{VP}_{3}$ muestra mayor homogeneidad asociando especies de requerimientos de hábitat complejos y de mayor conservación como como Tapirus terrestres y Lagothrix lagothricha.

\section{Discusión}

La riqueza de mamíferos hallada en el área de estudio es típica de la Amazonia colombiana, la mayoría representan especies endémicas, con hábitos generalistas, ubicadas en niveles tróficos medios (Defler 2004, Solari et al. 2013) cuantificada en 15 especies, de 45 que alberga el territorio (Ramírez-Cháves et al. 2016).

Fue notorio el grado de tolerancia a la perturbación de su hábitat. En contraste, en el BAT/PV $\mathrm{P}_{1}$, la relación entre Cheracebus torquatus, Sapajus apella y L. nigricollis revela una marcada sinantropia con un comportamiento agonístico y de forrajeo permi-

Tabla 2. Índices de diversidad del ensamble de mamíferos presentes en los parches de bosque de la inspección Los Pozos, San Vicente del Caguán, Caquetá, Colombia

\begin{tabular}{|c|c|c|c|c|}
\hline Índice & $\mathrm{BAT} / \mathrm{PV}_{1}$ & $\mathrm{BG} / \mathrm{P}_{2}$ & $\mathrm{BG} \mathrm{VP}_{3}$ & BAT/P $_{4}$ \\
\hline Taxa S & 12 & 7 & 11 & 7 \\
\hline Individuals & 38 & 20 & 28 & 19 \\
\hline Dominance D & 0,14 & 0,25 & 0,15 & 0,28 \\
\hline Simpson 1-D & 0,85 & 0,74 & 0,84 & 0,71 \\
\hline Shannon $\mathrm{H}$ & 2,14 & 1,59 & 2,11 & 1,55 \\
\hline Margalef & 3,02 & 2,00 & 3,00 & 2,03 \\
\hline Equitability J & 0,861 & 0,82 & 0,88 & 0,80 \\
\hline
\end{tabular}




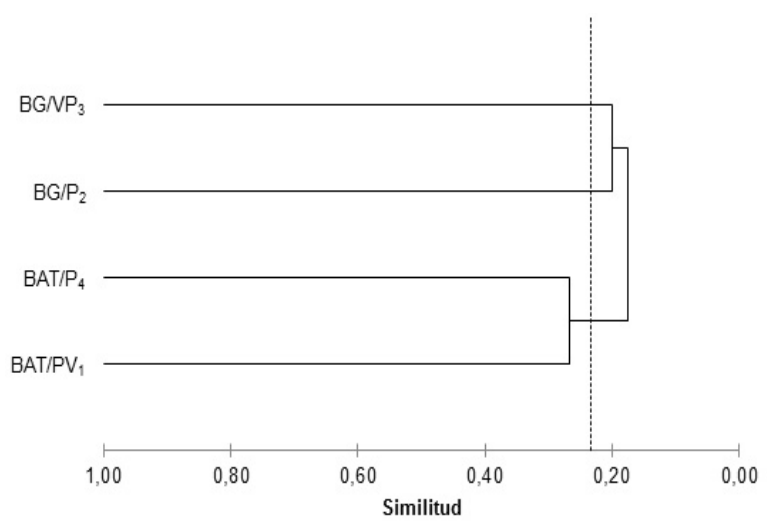

Figura 4. Dendrograma de similitud entre parches según las abundancias. pasar de un parche a otro.

Igualmente, los registros de Lagothrix lagothricha con crías en los parches $\mathrm{BAT} / \mathrm{PV}_{1}, \mathrm{BG} /$ $\mathrm{VP}_{3}$ y BAT $/ \mathrm{P}_{4}$, Alouatta seniculus en $\mathrm{BG} / \mathrm{P}_{2}$ y BG/ VP3 y Ateles belzebuth en $\mathrm{BAT} / \mathrm{PV}_{1}$ develan que estos remanentes de bosque constituyen una oferta para el mantenimiento de estas especies. Aunque $L$. lagothricha y A. belzebuth podrían desempeñar funciones como dispersores de semillas de alta calidad para el mantenimiento de estas áreas a largo plazo (Defler 2004, Rodríguez-M et al. 2006, Stevenson y Guzmán-Caro 2013), no existen garantías para que las poblaciones puedan mantenerse a futuro (Gómez-Posada et al. 2009) debido a los acelerados

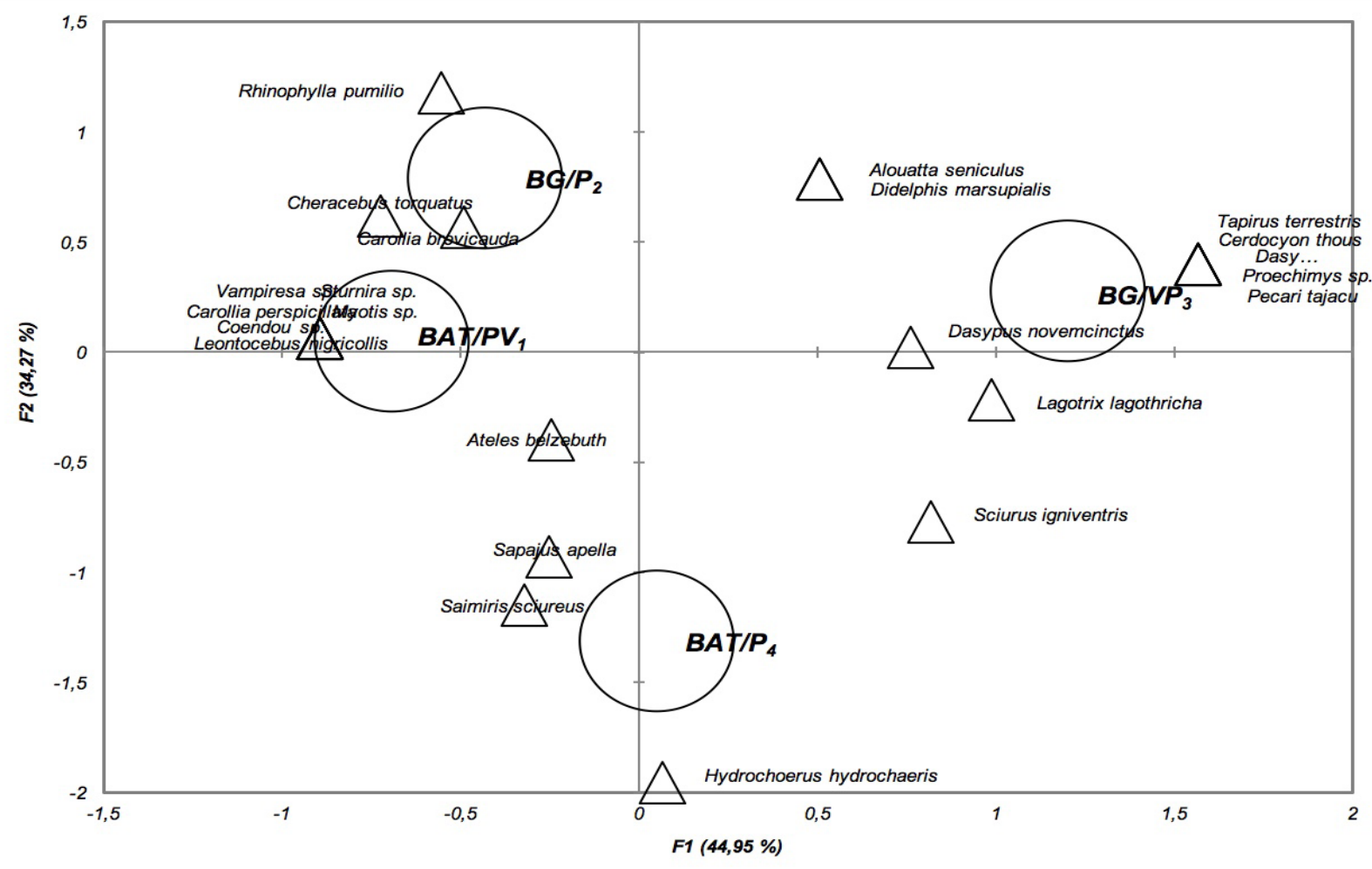

Figura 5. Análisis de correspondencia de las abundancias de las especies de mamíferos y los parches de bosque.

tiéndoles evitar la competencia utilizando diferentes estratos del bosque, favoreciéndose ya sea por retoños, flores, frutos y demás recursos disponibles. Este tipo de aprovechamientos eleva la importancia de estos relictos de bosque (Pérez Sánchez 2005) permitiendo las asociaciones interespecíficas (en el caso de los primates) (Echavez 2013). Sin embargo, son amenazadas principalmente por colisión de vehículos automotor en las vías debido al riesgo que asumen al descender hasta el suelo (en los arborícolas) para procesos de fragmentación.

La danta (T. terretris) se presentó solo en el BG/ $\mathrm{VP}_{3}$, características que coinciden con los requerimientos de hábitat (vegetación secundaria y bosques de galería) descritos por Arias-Alzate et al. (2009) y Gonzáles-D et al. (2014). Aparentemente T. terrestres cuenta con la disponibilidad de espacio y recursos, debido a la conectividad que existe entre el $\mathrm{BG} / \mathrm{VP}_{3}$ y el BAT $/ \mathrm{P}_{4}$, lo que posibilitaría la movilidad de la especie, sabiendo que esta recorre hasta $4.049 \mathrm{~km}$ 
Tabla 3. Valores de similitud en la composición de las especies

\begin{tabular}{|c|c|c|c|c|}
\hline Parche & $\mathrm{BAT} / \mathrm{PV}_{1}$ & $B G / P_{2}$ & $\mathrm{BG} \mathrm{VP}_{3}$ & BAT/P $_{4}$ \\
\hline BAT/PV & 6 & 0,188 & 0,15 & 0,26 \\
\hline $\mathrm{BG} / \mathrm{P}_{2}$ & 3 & 1 & 0,20 & 0,16 \\
\hline$B G /{ }^{2} P_{3}$ & 3 & 3 & 5 & 0,20 \\
\hline $\mathrm{BAT} / \mathrm{P}_{4}$ & 4 & 2 & 3 & 1 \\
\hline
\end{tabular}

Los valores superiores representan el grado de similitud entre los parches.

diarios en promedio (Gonzáles-D et al. 2014) y el $\mathrm{BG} / \mathrm{VP}_{3}$ posee $4 \mathrm{~km}^{2}$ aproximadamente.

En el caso de los quirópteros la composición de especies coincide con los listados presentados por Marín-Vásquez et al. (2005b), González-Reyes y Velásquez-Valencia (2011) y García-Cedeño et al. (2005). La presencia de Myotis sp. es posiblemente una muestra de su función como controladores poblacionales de pequeños y grandes insectos, de igual forma $C$. brevicauda y C. perspicilata representan una comunidad en constante intervención que se benefician de la apertura de nuevos hábitats y que encuentran recursos en zonas de regeneración temprana (Oria y Machado 2007). Sin embargo, el estudio de este grupo se ve limitado debido a conflictos de orden social en la región lo que impide los muestreos nocturnos en la zona. Teniendo en cuenta los listados nacionales (Solari et al. 2013) y las adiciones realizadas por Ramírez-Chávez et al. (2016) confirman que Caquetá alberga $23 \%$ de la diversidad nacional de murciélagos y siendo este grupo indispensable en la contribución de la regeneración de los bosques y por su papel como dispersores de semillas (Estrada-Villegas et al. 2007) se hace indispensable poder continuar con investigaciones que permitan tener aproximaciones más concretas al estado real de esta comunidad.

Conservación de mamíferos. Del total de especies encontradas, dos primates y la danta o tapir (Lagothrix lagothricha, Ateles belzebuth y Tapirus terrestres) representan un especial interés en temas de conservación para Colombia (Tabla 4) (CITES, UICN Versión 2016-2 y Res 192/2014).

En este enfoque, mediante el análisis de correspondencia (Figura 6) se encontró que la especie en peligro (EN) se asocia positivamente con $\mathrm{BAT} / \mathrm{P}_{4} \mathrm{y}$ las especies VU están asociadas positivamente con el BAT/ $\mathrm{P}_{3}$. Según los reportes de la UICN (2016-2) los primates son uno de los grupos taxonómicos más amenazados y llevados a la extinción, por esto se hace prioritario establecer acciones de conservación y manejo estricto en estas áreas, de manera que las poblaciones que aún existen puedan mantenerse en el tiempo.

Las principales amenazas para los mamíferos presentes en esta zona de estudio son las actividades humanas como la cacería, la extracción maderera y la explotación petrolera. La actividad maderera es más recurrente en los parches $\mathrm{BAT} / \mathrm{PV}_{1}$ y $\mathrm{BG} / \mathrm{P}_{2}$ representando por sí misma, una amenaza puntual sobre las poblaciones de mamíferos y en especial de los primates, que pueden experimentar disminuciones drásticas en sus poblaciones debido a la reducción y pérdida de sus hábitats. En la medida que se agoten los recursos y aumente la presión de caza, las poblaciones se verán alteradas al punto de generar extinciones locales.

La caza representa una presión esporádica en las especies de roedores y cingulatos medianos, como puercoespín (Coendou sp.), chigüiro (Hydrochoerus hydrochaeris), guatín (Dasyprocta sp.) y en especial de los armadillos (Dasypus novemcinctus) debido a la demanda de su carne.

\section{Conclusiones}

En general, el área de estudio alberga una alta diversidad de mamíferos, que corresponde al $10 \%$ de

Tabla 4. Categorías de amenaza de especies con especial interés para la conservación, encontradas en Los Pozos, San Vicente del Caguán, Colombia

\begin{tabular}{llccc}
\hline Nombre común & \multirow{2}{*}{ Especie } & \multicolumn{3}{c}{ Categorías de amenaza } \\
& & UICN & Res 192 & CITES \\
\hline Danta o tapir & Tapirus terrestris & VU & CR & II \\
Mono araña o marimonda & Ateles belzebuth & EN & VU & II \\
Churuco & Lagothrix lagothricha & VU & VU & II \\
\hline
\end{tabular}

CR: Peligro crítico de extinción, EN: En peligro de extinción, VU: Vulnerable 


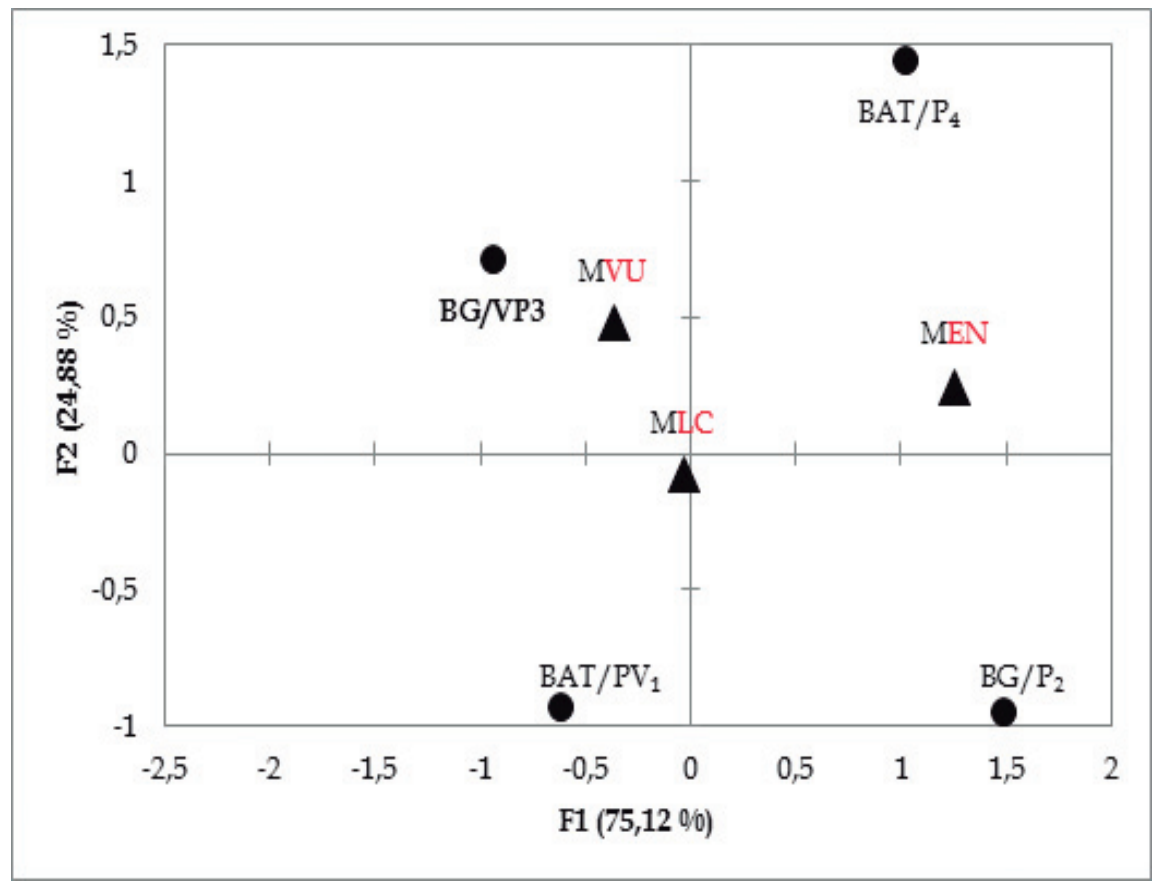

Figura 6. Análisis de correspondencia de las categorías de amenaza de la UICN de las especies vs. los parches del sitio de muestreos.

Mamíferos (M), Peligro crítico (MEN), Vulnerable (MVU), Preocupación menor (MLC)

la fauna estimada para la región amazónica colombiana y $4 \%$ para todo el territorio colombiano (Solari et al. 2013, Ramírez-Cháves y Suárez-Castro 2014, Ramírez-Cháves et al. 2016), sin incluir las posibles especies de roedores y pequeños marsupiales. Estas ofrecen servicios ecosistémicos (dispersores de semillas, controladores poblacionales de insectos y presas de caza) al igual que se comportan como especies bioindicadoras (Muzzachiodi y Sabattini, 2002) y aunque la fragmentación constituya en si una amenaza puntual para la mastofauna presente en la zona, este estudio revela que los relictos de bosque aun componen importantes zonas de refugios con reservas que ayudan al mantenimiento actual de las poblaciones.

De las 38 especies identificadas, se exalta la presencia de tres ubicadas en categorías de amenaza elevadas (EN, VU). Estas constituyen una reserva biológica importante en la región de la Amazonia Andina colombiana y ameritan un tratamiento urgente desde la óptica de la creación de áreas protegidas teniendo en cuenta que su distribución es exclusivamente amazónica y desempeñan funciones tróficas a escalas macroecológicas, que resultan indispensables en el funcionamiento de los ecosistemas.
Históricamente el departamento del Caquetá se ha visto presionado por conflictos de orden social, económico y cultural, lo que ha limitado el estudio de su biodiversidad, por esto se requiere un mayor esfuerzo de muestreo que permita la adición de especies a los listados y así tener un diagnóstico más fiable y completo para este grupo.

\section{Literatura citada}

Aguirre LF, Vargas A, Solari S. 2009. Clave de campo para la identificación de los murciélagos de Bolivia. Cochabamba: Centro de Estudios en Biología Teórica y Aplicada; 38 pp.

Arias Alzate A, Palacio Vieira JA, Muñoz-Durán J. 2009. Nuevos registros de distribución y oferta de hábitat de la Canta colombiana (Tapirus terrestris colombianus) en las tierras bajas del norte de la cordillera central (Colombia). Mastozool Neotrop. 16 (1): 19-26.

Boddicker M, Rodríguez JJ, Amanzo J. 2002. Indices for assessment and monitoring of large Mammals within an adaptive management framework. Environ Monit Assess. 76: 105-23. doi:10.1023/A:1015225022101

CITES. 2016. Comercialización de especies. Resolución Conf. 12.11 (Rev. CoP16). (en línea) (acceso 10 de agosto, 2016). Disponible en: http://checklist.cites.org/\#/es

Crump ML, Scott NG. 1994. Standart techniques for inventory and monitoring. En: Heyer MA, Donelly RW, McDiarmid LA, Hayek C. Foster MS (eds.). Measuring and monitoring 
biological. Standart methods for amphibians. Washington DC: Smithsonian Institution Press; pp. 77-171.

Defler TR. 2004. Primates de Colombia. Bogotá: Conservación Internacional Colombia; 543 pp.

Echavez Polo XA. 2013. Evaluación de la densidad poblacional de la comunidad de primates en áreas expuestas a diferente presión de cacería en la frontera amazónica entre Colombia y Perú. (Trabajo de Grado). Sincelejo: Facultad de Educación y Ciencias, Programa de Biología, Universidad de Sucre; $72 \mathrm{pp}$.

Estrada-Villegas E, Pérez-Torres J, Stevenson P. 2007. Dispersión de semillas por murciélagos en un borde de bosque montano. Ecotropicos. 20 (1): 1-14.

García-Cedeño Y, Velásquez-Valencia A, Marín-Vázques A, Cruz-Trujillo EJ, Aguilar-Gonzáles S, Malambo-Lozano C. 2005. Lista Anotada de Marsupiales (Mammalia: Didelphimorphia) del piedemonte Amazónico (Caquetá-Colombia). Momentos de Ciencia. 2 (1): 42-8.

Gómez-Posada C, Álvarez Z, Giraldo-Chavarriaga P. 2009. Densidad y estatus poblacional de monos aulladores rojos en un guadual, fragmento aislado, La Tebaida, Quindío, Colombia. Universitas Scientiarum. 14 (1): 8-15.

Gonzales-Reyes LJ, Velásquez-Valencia A. 2011. Composición de la comunidad de murciélagos en un área urbana del municipio de Florencia-Caquetá. Momentos de Ciencia. 8(2): 96-101.

González-D TM, Kaston Flórez F, Armentera D. 2014. Aportes al uso de coberturas de la danta de tierras bajas, Tapirus terrestris colombianus Hershkovitz 1954 (Perissodactyla: Tapiridae) en la sierra nevada de Santa Marta en las cuencas río Ancho y Palomino, Norte de Colombia. Bol Cient Mus Hist Nat U. de Caldas. 18 (1): 125-37.

IGAC. 1993. Aspectos ambientales para el ordenamiento territorial del occidente del departamento del Caquetá. Bogotá: Tercer Mundo Editores; 556 pp.

IGAC. 2014. Estudio general de suelos y zonificación de tierras departamento de Caquetá, escala 1.100.000. Bogotá: Instituto Geográfico Agustín Codazzi; 410 pp.

IUCN. 2016-2. The IUCN Red list of Threatened Species. (en línea). (acceso 10 agosto, 2016). Disponible en: http:// http://www.iucnredlist.org/search

Leite Pitman R. 2012. Mamíferos grandes de Loreto, Perú. Rapid Color Guide \# 287. Versión 2. Chicago: The Field Museum.

Marín-Vásquez A, Aguilar-González AV. 2005a. Murciélagos (Chiroptera) del departamento de Caquetá-Colombia. Biota Colomb. 6 (2): 211-8.
Marín-Vásquez A, Aguilar-González AV, Velásquez-Valencia A. 2005b. Murciélagos del Cerro Sinaí (Florencia-Caquetá). Momentos de Ciencia. 2 (1): 3-6.

Muzzachiodi N, Sabattini RA. 2002. La mastofauna como indicador de conservacion del bosque nativo en un área protegida de entre ríos. Rev Cient Agropec. 6: 5-15.

Oria FV, Machado MC. 2007. Determinación de la dieta de algunas especies de murciélagos (Mammalia: Chiroptera) de la cordillera central de Venezuela. FARAUTE Cien Tec. 2 (2): 5-15.

Pérez Sánchez J. 2005. Relaciones interespecíficas de un grupo de micos maiceros cariblancos Cebus albifrons versicolor durante los procesos de rehabilitación y libración. (Trabajo de grado). Bogotá: Facultad de Ciencias, Pontificia Universidad Javeriana; $155 \mathrm{pp}$.

Ramírez-Cháves HE, Suárez-Castro AF. 2014. Adiciones y cambios a la lista de mamíferos de Colombia: 500 especies registradas para el territorio nacional. Mammal Notes. 1 (2): $31-4$.

Ramírez-Cháves HE, Suárez-Castro AF, González-Maya JF. 2016. Cambios recientes a la lista de mamíferos de Colombia. Mammal Notes. 3 (1): 1-21.

Rodríguez MJV, Alberico M, Trujillo E, Jorgenson J (eds.). 2006. Libro Rojo de los Mamíferos de Colombia. Serie Libros Rojos de Especies Amenazadas de Colombia. Bogotá: Conservación Internacional Colombia \& Ministerio de Ambiente, Vivienda y Desarrollo Territorial; 433 pp.

Santos T, Tellería TL. 2006. Pérdida y fragmentación del hábitat: efecto sobre la conservación de las especies. Ecosistemas. 2: 3-12.

Solari S, Muñoz-Saba Y, Rodríguez-Mahecha JV, Defler TR, Ramírez-Chaves HE, Trujillo F. 2013. Riqueza, endemismo y conservación de los mamíferos de Colombia. Mastozool Neotrop. 20 (2): 301-65.

Stevenson PR, Guzmán-Caro DC. 2013. Procesos ecosistémicos provenientes del papel de los monos churucos (Lagothrix lagothricha) como dispersores de semillas. Pp. 332-47. Capítulo 21. En: Primates colombianos en peligro de extinción. Bogotá: Asociación Primatológica Colombiana; $348 \mathrm{pp}$.

Villarreal HM, Álvarez S, Córdoba F, Escobar G, Fagua F, Gast $\mathrm{H}$, et al. 2004. Manual de métodos para el desarrollo de inventarios de biodiversidad. Programa de inventarios de biodiversidad. Bogotá: Instituto de Investigación de Recursos Biológicos Alexander van Humboldt; 236 pp. 